\title{
Is a 'general' theory of addiction possible? A commentary on: a multistep general theory of transition to addiction
}

\author{
Aldo Badiani \\ Received: 20 January 2014 / Accepted: 23 February 2014 / Published online: 3 June 2014 \\ (C) Springer-Verlag Berlin Heidelberg 2014
}

Deroche-Gamonet and Piazza's thoughtful and ambitious paper contains much that can be agreed upon. By weaving into a single narrative, theories, models and experimental findings, its authors have provided the readers with a superb synopsis of much that has taken place in the field of addiction neuroscience over the last three decades.

This paper, however, is much more than a synopsis. As befitting to any major piece of scholarly work, it is a catalyst of ideas concerning the very nature of addiction, thus triggering a number of considerations, many more than can be crammed into a commentary. Out of these considerations, possible stumbling blocks for the present theory appear to emerge. I will limit myself to highlight three sets of issues that may be of general interest to researchers in the field, one set for each of the three sections of the paper.

\section{Defining addiction}

Herald: It is Othello's pleasure [...] that [...] every man put himself into triumph; some to dance, some to make bonfires, each man to what sport and revels his addiction leads him. (Shakespeare, The Tragedy of Othello, Moor of Venice, 2.2.1116-1120).

Any theory is critically dependent on a preliminary definition of its object, 'drug addiction' in this case. However,

\footnotetext{
A. Badiani

Department of Physiology and Pharmacology, Sapienza University, Rome, Italy

A. Badiani $(\triangle)$

School of Psychology, University of Sussex, Pevensey I, Room 2B19, BN1 9RH Brighton, UK

e-mail: aldo.badiani@uniroma1.it
}

Deroche-Gamonet and Piazza, decline to define addiction, in order 'to avoid a circular definition', and instead invite the readers to 'leave preconceptions aside and focus on the patient' (i.e., the addict). Yet, despite their best intentions, they immediately enter a circularity trap, as they base their definition of an addict on the Diagnostic and Statistical Manual of Mental Disorders (DSM) criteria for dependence (i.e. addiction). It would be difficult to think about a more circular definition than this. The notion of 'full addiction' appears often in the paper, but, again, without a clear-cut definition except for its equivalence to 'loss of control'. Thus, it is not clear what the operational definition of 'transition to addiction' is, as this would require the definition of start and end points.

In truth, defining addiction is not an easy task and still represents a considerable source of scholarly dispute. The term addiction has been and it is still used in at least three different ways. First, it is used as a lay term, which entered the English language in the late sixteenth century (maybe owing to Shakespeare) to indicate inclination or proclivity for certain habits or activities, in both its positive and negative connotations, including excessive drinking and smoking (Oxford English Dictionary 2010). Since the late nineteenth century, addiction is also used as a medical term to indicate pathological, compulsive drug use (e.g. Mattison 1892; Huntly 1896). Interestingly, the medical use of the term did not stop even when, in the mid-1960s, the term dependence replaced addiction in the eighth revision of the International Classification of Diseases of the World Health Organization (ICD-8, 1965) and in the second revision of the Diagnostic and Statistical Manual of the American Psychiatric Association (DSM-II, 1968), as well as in the denomination of the 14th WHO Expert Committee on Dependence-Producing Drugs (1965) versus the 13th WHO Expert Committee on Addiction-Producing Drugs (1964). Finally, addiction is used as a psychological construct to indicate a compulsive motivational drive (e.g. Robinson 
and Berridge 1993). Instances of all three meanings abound in the scholarly literature, sometimes within the same paper.

Here, Deroche-Gamonet and Piazza use addiction in its medical sense, with initial reference to the DSM-IV criteria for Substance Dependence distinct from Substance Abuse (American Psychiatric Association 1994). 'The distinction between abuse and dependence was based on the concept of abuse as a mild or early phase and dependence as the more severe manifestation' (American Psychiatric Association 2013a). This partition offered a ready-made diagnostic platform for developing bona fide animal models of drug addiction (e.g. Deroche-Gamonet et al. 2004; Vanderschuren and Everitt 2004) distinct from models of drug abuse.

Unfortunately, in following the DSM, Deroche-Gamonet and Piazza venture into dangerous terminological waters. The distinction between abuse and dependence is in fact completely lost with the DSM-5 (American Psychiatric Association 2013b), both conditions being subsumed under a unidimensional diagnostic label, Substance Use Disorders (SUDs), based on a set of 11 criteria. Much has been said about the pros and cons of this revision (e.g., O'Brien 2011, Uchtenhagen 2011; Hasin et al. 2013; Rehm et al. 2013). Certainly, one of its unintended consequences has been that of depriving pre-clinical researchers of an agreed set of clinical criteria for the definition of addiction. Alternatively, the entire spectrum of SUDs should be considered synonymous to addiction. As pointedly asked by Marcus Heilig (2011), 'why did the committee [i.e. the DSM-5 Substance-Related Disorders Work Group] stop short of simply calling the chapter "Addictive disorders"?' This, however, would still leave preclinical researchers without separate definitions for abuse and addiction.

The rationale for a single substance use disorder in DSM-5 was based, among other considerations, on the fact that 'common assumptions about the relationship of abuse and dependence were shown to be incorrect in several studies' (Hasin et al. 2013). Deroche-Gamonet and Piazza, however, turn this rationale on its head by suggesting that in DSM-IV, abuse and dependence corresponded to 'conceptually independent categories representing a different realm of problematic use' (if this were true, the same individual could receive both diagnoses, which the DSM-IV explicitly excluded) whereas in DSM5 abuse could be considered as a prodromal condition to dependence. They argue in fact (see Table 1 in DerocheGamonet and Piazza 2013) that DSM-5 mild (2-3 criteria out of 11) and moderate (4-5 criteria) SUDs correspond to DSM-IV substance abuse, whereas severe SUDs (6 or more criteria) are tantamount to DSM-IV substance dependence.

This simplification is not correct. In DSM-5, it is possible to meet the criteria for severe SUDs, without any of the old DSM-IV criteria for substance dependence. Indeed, the critical threshold of six criteria for severe SUDs can be reached by combining the three residual DSM-IV criteria for substance abuse (points 1-3 in Table 1), with 'tolerance' and 'withdrawal,, and with 'craving,' a novel, and much criticised criterion. On the other hand, the combination of diagnostic criteria 6,7 and 9 of Table 1 (Deroche-Gamonet and Piazza 2013) would correspond to a mild SUD in DSM-5 even if they would suffice for a diagnosis of substance dependence in DMS-IV. What would be the appropriate animal models for these two combinations of criteria? The fact is that in DSM- 5 , the diagnosis of mild, moderate and severe SUD can be based, even discarding tolerance and withdrawal, on hundreds of different combinations of criteria.

In summary, DSM-5 does not provide any easy solution to the problem of identifying different steps in the development of addiction, and therefore does not serve the purpose of the present theory. One way out of this terminological Gordian knot would be to dispense with DSM-5 altogether. It may be argued that Table 1 (Deroche-Gamonet and Piazza 2013) provides free-standing (even if loosely based on DSM-5) diagnostic criteria to identify the clinical entities labelled $\mathrm{ReS}, \mathrm{ISuE}$ and LoC. If this is the case, Deroche-Gamonet and Piazza should have stated it clearly, renouncing the protective umbrella of DSM. Whatever the approach used to define the three steps, it must have internal consistency, because, as noted above, definitions have critical implications for the identification of the appropriate animal models. It is difficult, for example, to understand why 'important social, work or recreational activities given up because of use' should be associated to LoC whereas 'Continued substance use despite persistent or recurrent social or interpersonal problems' should be associated to ISuE (in DSM-5, both criteria belong to the grouping of 'social impairment' and not of 'impaired control').

Finally, it should be noted that the Research Domain Criteria (RDoC) project cannot serve as a source of diagnostic criteria for addiction. As noted by Cuthbert and Insel (2013), 'Rather than starting with symptom-based definitions of disorders and working toward their pathophysiology, RDoC inverts this process. Basic science - in genetics, other areas of neuroscience and behavioural science - serves as the starting point, and disorders are considered in terms of disruptions of the normal-range operation of these systems, with an emphasis on the mechanisms that serve to result in dysfunctions of varying degrees.' That is, in the logic of the $\mathrm{RDoC}$ project, clinical definitions are an end point and not a starting point.

\section{How 'general' is the multistep general theory?}

Deroche-Gamonet and Piazza detail the scope of their paper at the very onset: 'This paper is not an extensive review of the literature on addiction but a position paper largely based on our own work and more generally on contributions of the 
Bordeaux School of Psychobiology.' Despite its obvious limitations, this declaration of intent would have had at least the advantage of clarity, if it were not flatly contradicted in the Conclusion by a much grander claim: '[...] this not just a theory but a general theory - the first of its kind in the field of addiction research. [...] General theories attempt to provide a framework that explains and integrates all existing relevant knowledge and previous existing theories. We believe that ours is a general theory of transition to addiction because it consolidates the knowledge generated by the drug addiction research field over 40 years of research.'

Certainly, this is not the first general theory of either addiction or transition to addiction (see, for example, West 2006 and West and Brown 2013). Furthermore, if the theory really aimed at encompassing all previous existing theories and models, it clearly fell short of this objective. Many theoretical models of addiction are not even taken into consideration. It suffices to say there are even theories that challenge altogether the notion of addiction as a psychiatric condition and that conceptualise it in terms of more or less rational choice (e.g. Heyman 2013).

Even if the scope of the Multistep General Theory were to be narrowed to neuroscience, there would still be some puzzling omissions. The decision to exclude the findings obtained with animal models of drug relapse, for example, is particularly disconcerting. Animal models of relapse are considered among the most heuristically relevant models of addiction, by definition, a chronic relapsing disorder and relapse is the single most important issue for treatment. In particular, the phenomenon of incubation (Pickens et al. 2011) appears to have important implications for the transition from drug use to addiction and as a target for therapy. Furthermore, the psychological and neurobiological processes underlying different causes of relapse appear be quite different from those responsible for the LoC stage, in which case, this omission becomes all the more striking.

The third and most important reason why the DerocheGamonet and Piazza's theory does not appear to be truly 'general' is that it lacks a critical prerequisite of any general theory of addiction, that is, its applicability to different addictive drugs. It is striking that the present theory is based almost exclusively on data obtained with cocaine or amphetamine (the word cocaine occurs 81 times whereas heroin only twice). Deroche-Gamonet and Piazza do hint at this problem, when they note that 'the shift to the LoC phase has only been studied with cocaine. It is then possible that the difficulty of increasing the number of subjects shifting to the LoC phase by modifying drug exposure, as described above, may not apply to other drugs.' Opiates and psychostimulants, however, have been shown to differ in many other important ways, including the role of dopaminergic system in drug taking (Ettenberg et al. 1982; Pettit et al. 1984); risk factors for transition to drug abuse in humans (Tsuang et al. 1998; Kendler et al. 2003) environmental influences on drug taking and drug preferences in human addicts and animals (Caprioli et al. 2008, 2009; Badiani and Spagnolo 2013) drug-induced synaptic plasticity in the ventral tegmental area (Dacher and Nugent 2011; Niehaus et al. 2010; Pan et al. 2008), nucleus accumbens (Robinson and Kolb 2004); prefrontal cortex (Huang et al. 2007; Lu et al. 2010; Van den Oever et al. 2008; Robinson and Kolb 2004), and neuroplastic changes related to incubation of drug craving (Pickens et al. 2011; Bossert et al. 2013). Further evidence of substance-specificity emerges when other addictive drugs are considered. In the case of alcohol, nicotine, benzodiazepines or barbiturates, for example, the mesocorticolimbic dopaminergic system appears to play a very different role, if any, relative to psychostimulants (e.g. Amit and Brown 1982, Rassnick et al. 1993, Laviolette and van der Kooy 2003).

To be fair, this criticism can be levied to the entire field of addiction neuroscience, which, in the last two decades, has mostly focused on the neuroadaptations induced by psychostimulant drugs and in particular by cocaine, seen as the prototypical, presumably representative addictive drug. It is, nevertheless, a criticism that should be of paramount concern for any 'general theory' of drug use, reward or addiction. It has been previously noted 'that generalisations from cocaine to other drugs of abuse should be made with extreme caution, and that the field would benefit from more systematic comparisons of the roles of different signalling molecules and synaptic plasticity mechanisms in reward and relapse across drug classes' (Badiani et al. 2011).

In summary, the viability of a general theory of addiction appears to be at odds with the growing attention to the substance-specific aspects of drug reward and drug addiction (e.g. Badiani et al. 2011, Peters et al. 2013, Bossert et al. 2013). In this respect, an interesting terminological change introduced by DSM-5 seems to have escaped the attention of most critiques. In the DSM-IV, Substance Abuse and Substance Dependence is used in the singular and is defined by a set of criteria independent of the specific substance. In the DSM-5, "each specific substance is addressed as a separate use disorder (e.g., alcohol use disorder, stimulant use disorder), although the diagnosis is based on the same overarching criteria" (American Psychiatric Association 2013a).

\section{Testing the multistep general theory}

Deroche-Gamonet and Piazza close their paper by describing the three features of their theory that are susceptible of validation. This section probably represents the less convincing part of the entire paper.

The first feature is: 'Transition to addiction depends on an interaction between individual vulnerabilities and drug 
exposure'. It is difficult to see who would disagree overall with this statement. Uncontroversial epidemiological data, duly cited by Deroche-Gamonet and Piazza, indicate that only a minority of drug users progress to problematic drug use, whereas the fulfilment of DSM criteria of SUD necessarily requires prolonged drug exposure. Most addiction theories incorporate, in one form or another, the notion of an 'interaction between individual vulnerabilities and drug exposure.' Indeed, it is difficult to see why the theories by Robinson and Berridge (1993) and by Koob and Le Moal (2008) are labelled as drug-centred, supposedly oblivious of individual differences in the vulnerability to addiction. The 1993 paper by Robinson and Berridge contains a section titled: 'Individual differences in the propensity to addiction', and Koob recently co-authored a paper titled 'Individual differences in prefrontal cortex function and the transition from drug use to drug dependence' (George and Koob 2010). Overall, this issue appears to be a classic 'straw man'.

The second feature is: 'Transition to addiction is a process that develops along at least three steps'. At a superficial level, also this statement appears to be quite uncontroversial (e.g. see Fig. 4 in Kalivas and Volkow 2005). Before receiving a diagnosis of severe alcohol use disorder (according to DSM-5), for example, an individual must have been a recreational drinker first, and then have gone through some intermediate stage. The problem is that, as discussed above, the Multistep General Theory does not provide any clear diagnostic definition of the three steps and thus of any way to test its validity. Parenthetically, it should be noted that Deroche-Gamonet and Piazza completely ignores other addiction-related phenomena, such as the so-called Dopamine Dysregulation Syndrome (Giovannoni et al. 2000; Pezzella et al. 2005; Merims and Giladi 2008), in which addiction-like behaviour is manifest in the absence of these three steps, seemingly falsifying their theory.

The third feature of the theory is: 'Transition to addition is a true psychiatric disease'. In all fairness, this feature appears to be little more than the rewording of the first one, as its falsification would depend on the "demonstration that in most conditions drug exposure is both necessary and sufficient to induce addiction.'

In summary, it is difficult to see in what way the testing of these features would distinguish the Multistep General Theory from other current theories of addiction. In contrast, it would be important to indicate how specific neurobiological elements of the theory may be validated. For example, as mentioned above, it would be important to verify whether the neurobiological processes underlying incubation of relapse (Pickens et al. 2011) are compatible with those responsible for the transition to the LoC stage.

\section{Conclusions}

Deroche-Gamonet and Piazza propose here a thoughtprovoking theory of addiction that nicely incorporates not only much of the work done by the Bordeaux group in the last three decades but also findings by other researchers in the field. The paper is ambitious and far-reaching in its implications. Yet, in stimulating the reader to ask questions that go beyond the theory itself and apply to the field as whole, this paper also underscores its major limitations. First, there is the issue of defining addiction and its stages so as to reflect clinical reality and, at the same time, to provide pre-clinical researchers with templates for animal models. Second, there is the issue of whether a truly general theory of addiction is possible. Until the 1980s, general theories of addiction were 'based on the common denominator of the so-called depressant drugs—opiates, barbiturates, alcohol and others' (Wise and Bozarth 1987). The major problem with these early attempts was the fact that psychostimulants 'did not readily fit models traditionally based on depressant drug classes.' The Psychomotor Stimulant Theory of Addiction by Wise and Bozarth (1987) opened a new phase in which general theories focused on psychostimulant drugs, and in particular, on cocaine. The emphasis on psychostimulants is now beginning to show its limits (Badiani et al. 2011, Peters et al. 2013, Bossert et al. 2013). The theory by Deroche-Gamonet and Piazza does not represent an exception to this rule, and therefore it does not appear to be as general as its title implies. Finally, in its present version the theory does not spell out criteria specific enough for its falsification.

It is certainly possible that the three stumbling blocks detailed above may be overcome in future developments of the Multistep General Theory, which will also prove whether a general theory of addiction is possible at all. Until then, the authors' farewell appears to be premature.

\section{References}

American Psychiatric Association (1994) Diagnostic and statistical manual of mental disorders, 4th ed (DSM-IV). The American Psychiatry Publishing, Washington

American Psychiatric Association (2013a) Substance use disorder fact sheet. www.dsm5.org/Documents/Substance Use Disorder Fact Sheet.pdf (accessed on January 14, 2014)

American Psychiatric Association (2013b) Diagnostic and statistical manual of mental disorders, 5th ed (DSM-5). The American Psychiatry Publishing, Arlington

Amit Z, Brown ZW (1982) Actions of drugs of abuse on brain reward systems: a reconsideration with specific attention to alcohol. Pharmacol Biochem Behav 17:233-238

Badiani A, Belin D, Epstein D, Calu D, Shaham Y (2011) Opiate versus psychostimulant addiction: the differences do matter. Nat Rev Neurosci 12:685-700 
Badiani A, Spagnolo PA (2013) Role of environmental factors in cocaine addiction. Curr Pharm Des 19:6996-7008

Bossert JM, Marchant NJ, Calu DJ, Shaham Y (2013) The reinstatement model of drug relapse: recent neurobiological findings, emerging research topics, and translational research. Psychopharmacol (Berl) 229:453-476

Caprioli D, Celentano M, Paolone G, Lucantonio F, Bari A, Nencini P, Badiani A (2008) Opposite environmental regulation of heroin and amphetamine self-administration in the rat. Psychopharmacol (Berl) 198:395-404

Caprioli D, Celentano M, Dubla A, Lucantonio F, Nencini P, Badiani A (2009) Ambience and drug choice: cocaine- and heroin-taking as a function of environmental context in humans and rats. Biol Psychiatry 65(10):893-899

Cuthbert BN, Insel TR (2013) Toward the future of psychiatric diagnosis: the seven pillars of RDoC. BMC Med 11:126. http://www. biomedcentral.com/1741-7015/11/126

Dacher M, Nugent FS (2011) Morphine-induced modulation of LTD at GABAergic synapses in the ventral tegmental area. Neuropharmacology 61:1166-1171

Deroche-Gamonet V, Belin D, Piazza PV (2004) Evidence for addictionlike behavior in the rat. Science 305:1014-1017

Ettenberg A, Pettit HO, Bloom FE, Koob GF (1982) Heroin and cocaine intravenous self-administration in rats: mediation by separate neural systems. Psychopharmacology (Berl) 78:204-209

George O, Koob GF (2010) Individual differences in prefrontal cortex function and the transition from drug use to drug dependence. Neurosci Biobehav Rev 35:232-247

Giovannoni G, O’Sullivan JD, Turner K, Manson AJ, Lees AJ (2000) Hedonistic homeostatic dysregulation in patients with Parkinson's disease on dopamine replacement therapies. J Neurol Neurosurg Psychiatry 68:423-428

Hasin DS, O'Brien CP, Auriacombe M, Borges G, Bucholz K, Budney A, Compton WM, Crowley T, Ling W, Petry NM, Schuckit M, Grant BF (2013) DSM-5 criteria for substance use disorders: recommendations and rationale. Am J Psychiatry 170:834-851

Heilig M (2011) A welcome change that stops short of being fully satisfying. Addiction 106:874-875

Heyman GM (2013) Addiction and choice: theory and new data. Front Psychiatry 2013 May 6;4:31. doi: 10.3389/fpsyt.2013.00031. eCollection 2013

Huang CC, Lin HJ, Hsu KS (2007) Repeated cocaine administration promotes long-term potentiation induction in rat medial prefrontal cortex. Cereb Cortex 17:1877-1888

Huntly WM (1896) Opium addiction: is it a disease? Proc of the Soc for the Study of Inebriety 50:1-12

Kalivas PW, Volkow ND (2005) The neural basis of addiction: a pathology of motivation and choice. Am J Psychiatry 162:1403-1413

Kendler KS, Jacobson KC, Prescott CA, Neale MC (2003) Specificity of genetic and environmental risk factors for use and abuse/dependence of cannabis, cocaine, hallucinogens, sedatives, stimulants, and opiates in male twins. Am J Psychiatry 160:687-95

Koob GF, Le Moal M (2008) Addiction and the brain antireward system. Annu Rev Psychol 59:29-53

Laviolette SR, van der Kooy D (2003) Blockade of mesolimbic dopamine transmission dramatically increases sensitivity to the rewarding effects of nicotine in the ventral tegmental area. Mol Psychiatry 8:50-59
Lu H, Cheng PL, Lim BK, Khoshnevisrad N, Poo MM (2010) Elevated BDNF after cocaine withdrawal facilitates LTP in medial prefrontal cortex by suppressing GABA inhibition. Neuron 67:821-833

Mattison JB (1892) Treatment of the morphine disease. Proc of the Soc for the Study of Inebriety 33:1-16

Merims D, Giladi N (2008) Dopamine dysregulation syndrome, addiction and behavioral changes in Parkinson's disease. Parkinsonism Relat Disord 14:273-280

Niehaus JL, Murali M, Kauer JA (2010) Drugs of abuse and stress impair LTP at inhibitory synapses in the ventral tegmental area. Eur J Neurosci 32:108-117

O'Brien C (2011) Addiction and dependence in DSM-V. Addiction 106 866-867

Oxford English Dictionary, 3rd ed (2010) Oxford University Press, Oxford, UK

Pan B, Hillard CJ, Liu QS (2008) Endocannabinoid signaling mediates cocaine-induced inhibitory synaptic plasticity in midbrain dopamine neurons. J Neurosci 28:1385-1397

Peters J, Pattij T, De Vries TJ (2013) Targeting cocaine versus heroin memories: divergent roles within ventromedial prefrontal cortex. Trends Pharmacol Sci 34:689-695

Pettit HO, Ettenberg A, Bloom FE, Koob GF (1984) Destruction of dopamine in the nucleus accumbens selectively attenuates cocaine but not heroin self-administration in rats. Psychopharmacology (Berl) 84:167-173

Pezzella FR, Colosimo C, Vanacore N, Di Rezze S, Chianese M, Fabbrini G, Meco G (2005) Prevalence and clinical features of hedonistic homeostatic dysregulation in Parkinson's disease. Mov Disord 20:77-81

Pickens CL, Airavaara M, Theberge F, Fanous S, Hope BT, Shaham Y (2011) Neurobiology of the incubation of drug craving. Trends Neurosci 34:411-420

Rassnick S, Stinus L, Koob GF (1993) The effects of 6 hydroxydopamine lesions of the nucleus accumbens and the mesolimbic dopamine system on oral self-administration of ethanol in the rat. Brain Res 623:16-24

Rehm J, Marmet S, Anderson P, Gual A, Kraus L, Nutt DJ, Room R, Samokhvalov AV, Scafato E, Trapencieris M, Wiers RW, Gmel G (2013) Defining substance use disorders: do we really need more than heavy use? Alcohol Alcohol 48:633-440

Robinson TE, Berridge KC (1993) The neural basis of drug craving: an incentive-sensitization theory of addiction. Brain Res Brain Res Rev 18:247-291

Robinson TE, Kolb B (2004) Structural plasticity associated with exposure to drugs of abuse. Neuropharmacology 47(Suppl 1):33-46

Tsuang MT, Lyons MJ, Meyer JM, Doyle T, Eisen SA, Goldberg J, True W, Lin N, Toomey R, Eaves L (1998) Co-occurrence of abuse of different drugs in men: the role of drug-specific and shared vulnerabilities. Arch Gen Psychiatry 55:967-72

Uchtenhagen A (2011) New and remaining problems with DSM-V. Addiction 106:888-889

Van den Oever MC, Goriounova NA, Li KW, Van der Schors RC, Binnekade R, Schoffelmeer AN, Mansvelder HD, Smit AB, Spijker S, De Vries TJ (2008) Prefrontal cortex AMPA receptor plasticity is crucial for cue-induced relapse to heroin-seeking. Nature Neurosci 11:1053-1058

Vanderschuren LJ, Everitt BJ (2004) Drug seeking becomes compulsive after prolonged cocaine self-administration. Science 305:1017-1019

West R (2006) Theory of addiction. Wiley, Oxford

West R, Brown J (2013) Theory of addiction. Wiley, Oxford

Wise RA, Bozarth MA (1987) A psychomotor stimulant theory of addiction. Psychol Rev 94:469-492 